\title{
A copula model for marked point processes
}

\author{
LIQUN DIAO \\ Department of Statistics and Actuarial Science, \\ University of Waterloo, Waterloo, ON, N2L 3G1, Canada \\ E-mail:12diao@uwaterloo.ca \\ RICHARD J. COOK \\ Department of Statistics and Actuarial Science, \\ University of Waterloo, Waterloo, ON, N2L 3G1, Canada \\ E-mail:rjcook@uwaterloo.ca \\ KER-AI LEE \\ Department of Statistics and Actuarial Science, \\ University of Waterloo, Waterloo, ON, N2L 3G1, Canada \\ E-mail:ka2lee@uwaterloo.ca
}

\begin{abstract}
Summary
Many chronic diseases feature recurring clinically important events. In addition, however, there often exists a random variable which is realized upon the occurrence of each event reflecting the severity of the event, a cost associated with it, or possibly a short term response indicating the effect of a therapeutic intervention. We describe a novel model for a marked point process which incorporates a dependence between continuous marks and the event process through the use of a copula function. The copula formulation ensures that event times can be modeled by any intensity function for point processes, and any multivariate model can be specified for the continuous marks. The relative efficiency of joint versus separate analyses of the event times and the marks is examined through simulation under random censoring. An application to data from a recent trial in transfusion medicine is given for illustration.
\end{abstract}

Keywords: Copula function, joint analysis, marks, recurrent events

The final publication is available at Springer via http://dx.doi.org/10.1007/s10985-013-9259-3.

\section{INTRODUCTION}

\subsection{OVERVIEW}

Many disease processes feature recurrent events which represent acute exacerbations of an underlying chronic condition. Examples include respiratory attacks in patients with asthma which can be associated with considerable disability and increased risk of death (Verona et al., 2003), flares of symptoms in patients with systemic lupus erythematosus (Petri et al., 1991; Fok et al., 2012), recurrent headaches 
among migraineurs (Pascual et al., 2000), and graft rejection episodes arising in transplant recipients where the rejection episodes signal increased risk of total graft rejection (Cole et al., 1994).

Statistical methods for the analysis of recurrent events have seen considerable development in the last three decades. The three primary classes of methods are based on intensity-based models (Andersen et al., 1993; Aalen et al., 2008), random effect models (Lawless, 1987a) and marginal models (Lawless and Nadeau, 1995). In the clinical trial arena, marginal methods based on rate functions (Andersen and Gill, 1982) have considerable appeal, and the development of methods for robust inference (Lawless and Nadeau, 1995) has led to their widespread use. Partially conditional models (Prentice, Williams and Peterson, 1981) and marginal methods based on multivariate failure time data (Wei, Lin and Weissfeld, 1989) are also used routinely.

The events in many conditions are severe enough to warrant therapeutic intervention for alleviation of symptoms and mitigation of risk for more serious complications. When such interventions are applied, there is typically a short-term response which reflects how effective the intervention was in alleviating symptoms and improving health. Studies of short-acting $\beta_{2}$-agonists for the treatment of asthma attacks (Sears et al., 1990), for example, aim to quickly improve lung function as measured by short term change in forced expiratory volume.

The data resulting from such processes feature event times, with each event having an accompanying attribute realized upon event occurrence. Marked point processes are suitable for modeling such data, and have been used extensively in areas such as seismology (Holden et al., 2002), genetics (Robin, 2002), image analysis (Descombes and Zerubia, 2002), insurance (Grandell, 1997, Ch. 9), finance (Prigent, 2001), forestry (Penttinen et al., 1992), and management science (Chen and Zheng, 1997). The theory of marked point processes is given in several excellent books on stochastic processes including Cox and Isham (1980, Chap. 5), Snyder and Miller (1991; Chap. 4), Karr (1991, Sect. 1.4), and more recently Daley and Vere-Jones (2008, Sect. 13.4). Semiparametric methods of analysis based on likelihood are discussed in Andersen et al. (1993, Sect. 2.4) and robust nonparametric marginal methods are considered in Cook et al. (2003) and Cook and Lawless (2007). Goulard et al. (1996) consider pseudo-likelihood methods and moment estimation is developed by Politis and Sherman (2001). The marks are often assumed to be independent of the event times to facilitate their separate modeling using simple methods, but this assumption is often questionable. Tests of the independence between the marks and event times were developed by Schlather et al. (2004), Schoenberg (2004), and Guan (2006).

We propose a novel model for a marked point process in which the marginal models for the events and the marks are compatible with standard models for recurrent event and longitudinal analyses. A copula function is used to link the "survival" function of each mark given the history of marks, with the distribution of the inter-event times given the relevant event history. This appealing structure means that analyses of the incidence of the events and analysis of the marks are compatible with standard methods for recurrent event data and any continuous multivariate distribution; efficiency gains can therefore be explored for joint versus separate analyses. The remainder of this paper is organized as follows. In Section ?? we define notation, describe the formulation of a copula-based marked point process model and construct the likelihood. Simulation studies and an illustration of the Mirasol platelet transfusion trial are presented in Sections ?? and ??, respectively, and general remarks and topics for future research are in Section ??.

\subsection{A Trial of Pathogen-Inactivated Platelets in Thrombocytopenic Patients}

Mirasol is a pathogen inactivation technology which utilizes exposure to ultraviolet light to inhibit the proliferation of pathogens and white blood cell replication to produce pathogen reduced platelets (PRT-PLT). The Mirasol Study is a recent multicentre trial of 118 hematology/oncology patients with chemotherapy induced thrombocytopenia reported in Cazenave et al. (2010). Patients were randomized to receive either pathogen reduced platelets (PRT-PLT) or standard platelets (Reference) 
as required over a 28 day treatment period. The primary outcome in this trial was based on the corrected count increment (CCI), which is the difference between the patients platelet count before and after the transfusion, adjusted for dose of platelets and body surface area of the patient (Davis et al., 1999). Patients receive prophylatic transfusions whenever their platelet counts drop below the defined threshold of $10 \times 10^{9} \mathrm{~m}^{2} / \ell$. As a result, one might expect an association between the CCI for a particular transfusion and the time to the next transfusion.
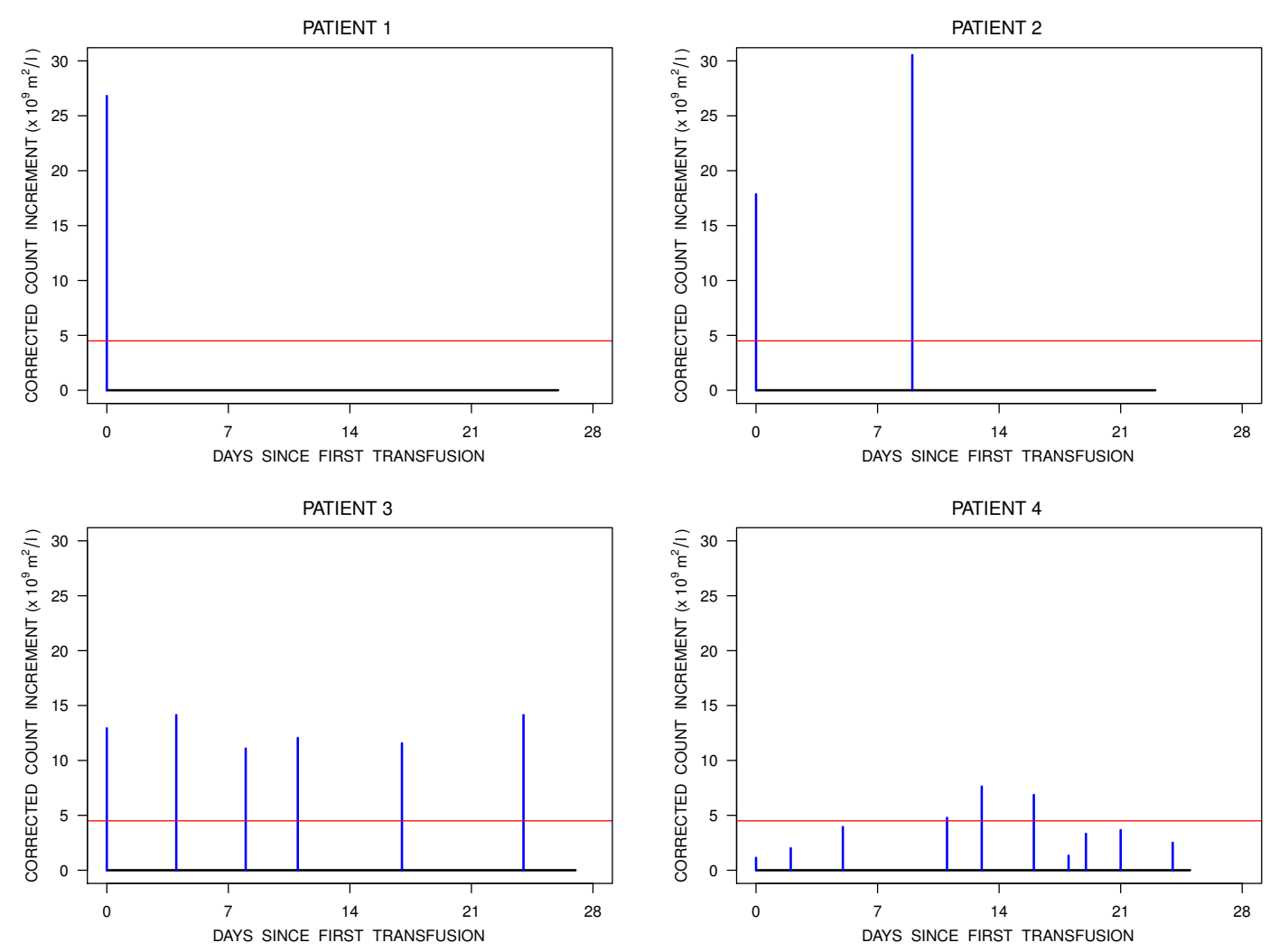

Figure 1: Profiles of four patients from the Mirasol Study (Cazenave et al., 2010) showing the times of the transfusions, the corrected count increments and the duration of follow-up; the horizontal line at $4.5\left(\times 10^{9} \mathrm{~m}^{2} / \ell\right)$ is the threshold for a successful transfusion.

This phenomenon is illustrated in Figure ?? which displays data for a sample of four patients from the Mirasol Study. In each panel, the horizontal axis indicates the number of days since the first transfusion and the vertical axis is the corrected count increment based on the measurement $24 \mathrm{~h}$ after the transfusion. The horizontal dashed line at 4.5 corresponds to the threshold used to define a successful $(\mathrm{CCI}>4.5)$ or unsuccessful $(\mathrm{CCI} \leq 4.5)$ transfusion. Patient 1 required only one transfusion during the 28 day treatment period and experienced a large increase in their platelet count due to this transfusion. Patient 4 on the other hand, required 9 transfusions and with only three of these leading to a successful response. Patients 2 and 3 had intermediate numbers of transfusions which generally lead to successful responses.

\section{MOdel Formulation AND LiKeliHOOd CONSTRUCTION}

\subsection{A Copula-Based Marked Point Process Model}

Let $X=1$ if the individual is randomized to receive the experimental intervention and $X=0$ otherwise. We consider studies in which the primary purpose is to compare the effect of the experi- 
mental intervention to the standard intervention with respect to the distribution of the responses, and secondary interest lies in the effect of the randomized intervention on event occurrence.

We suppose the process begins with an initiating event at $T_{0}=0$, let $T_{k}$ be the time of the $k$ th recurrence, and let $W_{k}=T_{k}-T_{k-1}$ denote the waiting time between the $(k-1)$ st and $k$ th event, $k=1, \ldots$. We let $N(t)=\sum_{k=1}^{\infty} I\left(T_{k} \leq t\right)$, where $I(\cdot)$ is the indicator function such that $I(A)=1$ if $A$ is true and $I(A)=0$ otherwise. The associated right-continuous counting process is $\{N(s), 0<s\}$ and the associated history is $H^{N}(t)=\{N(s), 0<s<t\}$. Let $\Delta N(t)=N\left((t+\Delta t)^{-}\right)-N\left(t^{-}\right)$ be the number of events over $[t, t+\Delta t), d N(t)=\lim _{\Delta t \rightarrow 0} \Delta N(t)=1$ if an event occurs at time $t$, and $d N(t)=0$ otherwise. The mark associated with the $k$ th event is denoted by the random variable $Y_{k}, k=0,1, \ldots$, and we let $Y(t)=\left\{Y_{0}, \ldots, Y_{N(t)}\right\}$ denote the set of marks realized over $[0, t]$. It is convenient to denote the history of the marks at $t$ as $H^{Y}(t)=\{Y(s), 0<s<t\}$, and the full history is then $H(t)=\{N(s), Y(s), 0<s<t, X\}$. Let $\left[0, C_{A}\right]$ denote the planned period of observation, where $C_{A}>0$ is an administrative right censoring time. Suppose further that $C_{R}>0$ is a random right-censoring time (Kalbfleish and Prentice, 2002; Lawless, 2003) giving a net duration of observation $C=\min \left(C_{A}, C_{R}\right)$; Figure ?? gives a schematic diagram relating key variables.

We let $C(t)=I(t \leq C)$ indicate whether the process is under observation at time $t$. Then if $d \bar{N}(t)=C(t) d N(t), d \bar{N}(t)=1$ implies that an event occurs and is observed at $t$. We define $\bar{N}(t)=\int_{0}^{t} d \bar{N}(s)$ and let $\bar{Y}(t)=\left\{Y_{0}, \ldots, Y_{\bar{N}(t)}\right\}$ denote the number of events and the respective marks observed over $(0, t]$. The corresponding histories at time $t$ are then $\bar{H}^{N}(t)=\{\bar{N}(s), C(s), 0<$ $s<t\}$ and $\bar{H}^{Y}(t)=\{\bar{Y}(s), C(s), 0<s<t\}$ and the full history is $\bar{H}(t)=\{\bar{N}(s), \bar{Y}(s), C(s), 0<$ $s<t, X\}$.

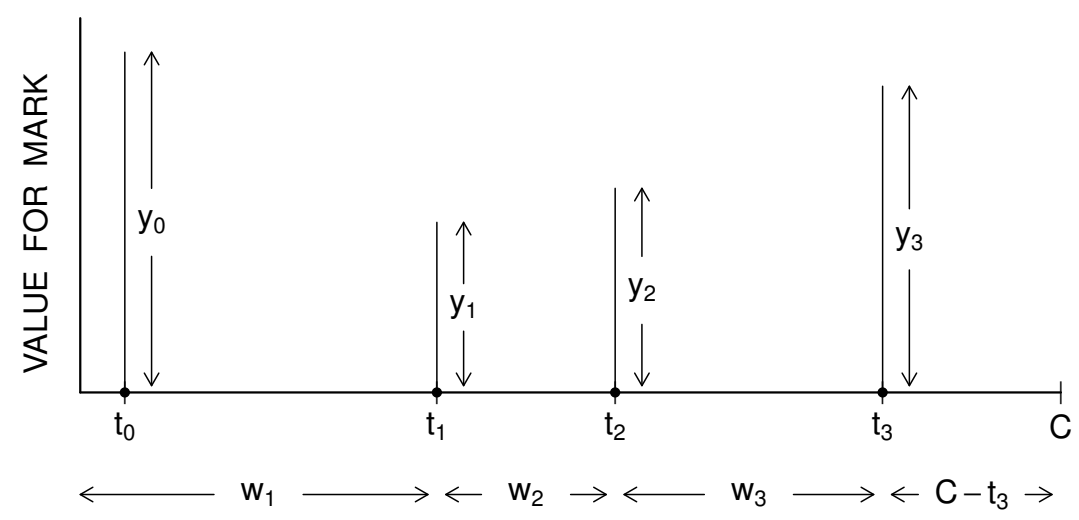

Figure 2: Schematic diagram with notation for a marked point process

The full likelihood for a marked point process observed over $\left(0, C_{A}\right]$ subject to random right censoring can be expressed in product integral notation (Cook and Lawless, 2007; Chap. 8) as

$$
\begin{aligned}
& \prod_{s \in\left[0, C_{A}\right]} P(C(s) \mid \bar{H}(s))\{P(d \bar{N}(s) \mid \bar{H}(s), C(s)=1) \\
&\left.P\left(Y_{\bar{N}(s)} \mid \bar{H}(s), C(s)=1, d \bar{N}(s)=1\right)^{d \bar{N}(s)}\right\}^{C(s)},
\end{aligned}
$$

where we informally let the conditional probability involving $Y_{\bar{N}(t)}$ represent a conditional density when the marks are continuous. Under non-informative censoring we omit the term $P(C(s) \mid \bar{H}(s))$ 
and work with the partial likelihood

$$
\begin{aligned}
& \prod_{s \in\left[0, C_{A}\right]}\{P(d \bar{N}(s) \mid \bar{H}(s), C(s)=1) \\
& \left.\quad P\left(Y_{\bar{N}(s)} \mid \bar{H}(s), C(s)=1, d \bar{N}(s)=1\right)^{d \bar{N}(s)}\right\}^{C(s)} .
\end{aligned}
$$

If censoring is conditionally independent, (??) is equal to

$$
\prod_{s \in\left[0, C_{A}\right]} P(d N(s) \mid H(s), C(S)) \prod_{s \in\left[0, C_{A}\right]} P\left(Y_{N(s)} \mid H(s), d N(s)=1\right)^{d \bar{N}(s)}
$$

and we can express the partial likelihood in terms of the model of interest.

We aim to specify a model such that the event intensity is formulated so that a marginal analysis of the recurrent event process yields parameters compatible with a standard recurrent event model while at the same time there is a dependence on the previous mark. Copula models can be used for this purpose (Joe, 1997; Nelsen, 2006) and we provide a brief general discussion of them first.

If $U_{1}$ and $U_{2}$ are two random variables with standard uniform marginals on $[0,1]$, the bivariate distribution

$$
\mathcal{C}\left(u_{1}, u_{2}\right)=P\left(U_{1} \leq u_{1}, U_{2} \leq u_{2}\right),
$$

corresponds to a copula function (Genest and MacKay, 1986). For two continuous random variables $V_{1}$ and $V_{2}$ with marginal survivor functions $\mathcal{F}_{j}(\cdot), j=1,2$, a joint survivor function $\mathcal{F}\left(v_{1}, v_{2}\right)=$ $P\left(V_{1} \geq v_{1}, V_{2} \geq v_{2}\right)$ is constructed through a copula by defining

$$
\mathcal{F}\left(v_{1}, v_{2}\right)=\mathcal{C}\left(\mathcal{F}_{1}\left(v_{1}\right), \mathcal{F}_{2}\left(v_{2}\right)\right) ;
$$

Sklar's theorem (Nelsen, 2006) ensures the existence and uniqueness of $\mathcal{C}:[0,1]^{2} \rightarrow[0,1]$. Suppose $\mathcal{F}_{j}\left(v_{j} \mid X\right)$ is the survivor function of $V_{j}$ given $X=x, j=1,2$. Patten (2006) proved for conditional distributions that for each $X=x$ in the support of $X$, the joint distribution of $V_{1}$ and $V_{2}$ given $X=x$ is uniquely defined by

$$
\mathcal{F}\left(v_{1}, v_{2} \mid x\right)=P\left(V_{1} \geq v_{1}, V_{2} \geq v_{2} \mid X=x\right)=\mathcal{C}\left(\mathcal{F}_{1}\left(v_{1} \mid x\right), \mathcal{F}_{2}\left(v_{2} \mid z\right) \mid x\right),
$$

for all $\left(v_{1}, v_{2}\right) \in \mathbb{R}^{2}$.

Returning now to the marked point process setting, we consider the case where the marks are continuous random variables and for convenience let $H_{k}^{N}=\left\{T_{1}, \ldots, T_{k-1}\right\}, H_{k}^{Y}=\left\{Y_{0}, \ldots, Y_{k-1}\right\}$ and $H_{k}=\left\{T_{1}, \ldots, T_{k-1}, Y_{0}, \ldots, Y_{k-1}, X\right\}$. If we let $\bar{N}(C)=n$, note that

$$
\prod_{s \in\left[0, C_{A}\right]} P(d N(s) \mid H(s), C(s))=\prod_{k=1}^{n} f_{k}\left(t_{k} \mid H_{k}\right) \cdot \mathcal{F}_{n+1}\left(C \mid H_{n+1}\right) .
$$

The joint distribution of $\mathcal{F}\left(T_{k}, Y_{k-1} \mid H_{k}^{N}, H_{k-1}^{Y}, X\right)$ can be defined through a copula $\mathcal{C}$ as

$$
\mathcal{C}\left(\mathcal{F}\left(t_{k} \mid H_{k}^{N}, H_{k-1}^{Y}, X\right), \mathcal{F}\left(y_{k-1} \mid H_{k}^{N}, H_{k-1}^{Y}, X\right) ; \phi\right),
$$

where $\phi$ is a vector of association parameters. This particular way of modeling the association between the marks and the events was chosen because in our motivating study, larger realized values of $Y_{k-1}$ are expected to lead to larger gaps $W_{k}=T_{k}-T_{k-1}$. To retain the feature that the marginal models for the event times and marks have a standard form, we make the following two assumptions:

Assumption A1: $\quad T_{k} \perp H_{k-1}^{Y} \mid H_{k}^{N}, X, k=1,2, \ldots$

Assumption A2: $\quad Y_{k-1} \perp H_{k}^{N} \mid H_{k-1}^{Y}, X, k=2,3, \ldots$ 
Assumption A1 means that given the history of the event times and the fixed covariate the marks at times prior to $T_{k-1}$ are not associated with $W_{k}=T_{k}-T_{k-1}$. Assumption A2 states that the mark at $T_{k-1}$ is independent of the event times prior to $T_{k}$ given the previous marks and the fixed covariate. This means that any models for longitudinal data can be used for the joint distribution of the marks. Under assumptions $\mathrm{A} 1$ and A2, (??) simplifies to involve $\mathcal{F}\left(t_{k} \mid H_{k}^{N}, X\right)$ (under A1) and $\mathcal{F}\left(y_{k-1} \mid H_{k-1}^{Y}, X\right)$, the "survivor function" of the mark given the history of the marks (under A2). By applying the copula constructed in (??) with assumptions A1 and A2 to (??), $\prod_{s \in[0, \infty)} P(d N(s) \mid H(s))$ becomes

$$
\prod_{k} f\left(t_{k} \mid H_{k}^{N}, X\right) \cdot c\left(\mathcal{F}\left(t_{k} \mid H_{k}^{N}, X\right), \mathcal{F}\left(y_{k-1} \mid H_{k-1}^{Y}, X\right)\right)
$$

where $c(\cdot, \cdot)$ is the density function of the copula $\mathcal{C}$ in (??).

The term $P\left(Y_{N(s)} \mid H(s), d N(s)=1\right)$ in the second part of the product integrand in (??) is the density of the mark at time $s$ conditional on the full history $H(s)$ and the fact that an event occurred at time $s$. It can be written in terms of event times and marks as $f\left(y_{k} \mid H_{k+1}^{N}, H_{k}^{Y}, X\right)$, and by assumption A2, this becomes

$$
P\left(Y_{N(s)} \mid H(s), d N(s)=1\right)=f\left(y_{k} \mid H_{k}^{Y}, X\right) .
$$

We have adopted this model formulation to ensure the marginal model for the event process can be any point process model and the marks can be modelled separately using any model for longitudinal data; the association model linking these two processes is based on a copula.

If $n$ events are observed for one individual over $(0, C]$, the likelihood of the observed outcome " $n$ events occur at times $t_{1}<\cdots<t_{n}$ with respective marks $y_{0}, y_{1}, \ldots, y_{n}$ given covariate $x$ " is proportional to

$$
\begin{aligned}
& \left\{\mathcal{C}^{(01)}\left(P\left(T_{n+1}>C \mid H_{n+1}^{N}, X\right), P\left(Y_{n}>y_{n} \mid H_{n}^{Y}, X\right)\right)\right. \\
& \left.\prod_{k=1}^{n} c\left(P\left(T_{k}>t_{k} \mid H_{k}^{N}, X\right), P\left(Y_{k-1}>y_{k-1} \mid H_{k-1}^{Y}, X\right)\right)\right\} \\
& \cdot\left\{\prod_{k=1}^{n} P\left(T_{k} \in\left[t_{k}, t_{k}+d t_{k}\right) \mid H_{k}^{N}, X\right)\right\} \cdot\left\{\prod_{k=0}^{n-1} P\left(y_{k} \in\left[y_{k}, y_{k}+d y_{k}\right) \mid H_{k}^{Y}, X\right)\right\}
\end{aligned}
$$

where $\mathcal{C}^{(01)}(u, v)=\partial \mathcal{C}(u, v) / \partial v$. The likelihood (??) is in a very amenable form. The first $n+1$ components provide information about all parameters; the second $n$ components provide information about the marginal model for the recurrent event; and the last $n+1$ components relate to the marginal model for the marks.

The formulation of this model, through the use of copula function, enables specification of the marginal and association models separately. Particular models for the recurrent events will be proposed in Section ?? and those for the marks in Section ??.

\subsection{Marginal Models for the Recurrent Events}

The "marginal" intensity function of the recurrent event process is defined as

$$
\lambda\left(t \mid H^{N}(t), X\right)=\lim _{\Delta t \rightarrow 0} \frac{P\left(\Delta N(t)=1 \mid H^{N}(t), X\right)}{\Delta t},
$$

where we assume that two events cannot occur at the same time. 


\subsubsection{MARKOV MODELS}

One might adopt a Markov model of the form

$$
\lambda\left(t \mid H^{N}(t), X\right)=\lambda_{k}(t \mid X),
$$

where $N\left(t^{-}\right)=k$, whereby the transition intensity depends on cumulative number of events over $(0, t]$; see Prentice et al. (1981). The Poisson intensity (Lawless, 1987b) arises if we set $\lambda_{k}(t \mid X)=$ $\lambda(t \mid X)$ for $k=0,1, \ldots$. Models with multiplicative covariate effects of the form

$$
\lambda(t \mid X ; \theta)=\lambda_{0}(t ; \alpha) \exp (X \beta),
$$

are very common, where $\lambda_{0}(t \mid X ; \alpha)$ is a baseline intensity (rate) function indexed by $\alpha$, and $\beta$ is a regression coefficient.

\subsubsection{A MiXed MARKov MOdel}

To allow for extra-Poisson variation, one can consider a standard mixed Poisson model, in which conditional on a random effect $U>0$ with $E(U)=1$ and $\operatorname{Var}(U)=\gamma$, the marginal intensity given $U$ is of the form

$$
\lambda\left(t \mid H^{N}(t), X, U\right)=\lim _{\Delta t \rightarrow 0} \frac{P(\Delta N(t)=1 \mid X, U)}{\Delta t}=U \lambda_{0}(t ; \alpha) \exp (X \beta) .
$$

Under this mixed Poisson model, if we define $E(N(t) \mid X)=\mu(t \mid X)$, then $\operatorname{Var}(N(t) \mid X)=\mu(t \mid X)+$ $\mu(t \mid X)^{2} \gamma$ and it is apparent that the mixed Poisson model accommodates extra Poisson variation. When $U$ follows a gamma distribution, the marginal intensity function becomes

$$
\left(\frac{1+N\left(t^{-}\right) \gamma}{1+\mu(t \mid X) \gamma}\right) \lambda_{0}(t ; \alpha) \exp (X \beta)
$$

which corresponds to a negative binomial process.

\subsubsection{A SEMI-MARKov MODEL}

Semi-Markov models are useful when there is a sort of renewal in the process when an event occurs. These feature intensities of the form

$$
\lambda\left(t \mid H^{N}(t), X\right)=h_{k}(B(t) \mid X),
$$

where $N\left(t^{-}\right)=k$ and $B(t)=t-T_{N\left(t^{-}\right)}$is the time since the most recent event. A renewal process is obtained if $h_{k}(s)=h(s)$ for $k=0,1, \ldots$ in which case the waiting times are independent and identically distributed within subjects. Use of mixed semi-Markov models is likewise possible, where given a random effect $U$ we may have

$$
\lambda\left(t \mid H^{N}(t), X, U\right)=U h_{k}(B(t) \mid X) .
$$

\subsection{Marginal Models For the Marks}

Let $Y=\left(Y_{0}, Y_{1}, \ldots, Y_{K}\right)^{\prime}$ denote the vector of $K+1$ marks. We may assume, for example, that $Y \mid X$ is a $(K+1) \times 1$ multivariate normal random variable with mean $\mu(X)=\left(\mu_{0}(X), \ldots, \mu_{K}(X)\right)^{\prime}$ and $(K+1) \times(K+1)$ covariance matrix $\Sigma$ with diagonal events $\sigma^{2}$ and off diagonal elements $\rho \sigma^{2}$. We may specify, for example, that $\mu_{k}(X)=\eta_{0 k}+\eta_{1 k} X$ and so the effect of treatment is to change the mean mark. Often one would set $\eta_{1 k}=\eta_{1}, k=1, \ldots$, to obtain a parsimonious representation of the treatment effect, although tests of $H_{0}: \eta_{1 k}=\eta_{1}$ are often sensible. When marks are binary, analogous multivariate binary models may be adopted and often these would be most naturally formulated with marginal specifications of treatment and other covariate effects, particularly for data arising in clinical trials. 


\subsection{ESTIMATION AND STATISTICAL INFERENCE}

Let $L(\theta ; z)$ denote the likelihood function (??), where $z$ denotes the data comprised of the marks $y_{0}, y_{1}, \ldots, y_{n}$ observed at time points $t_{1}, \ldots, t_{n}$, the right censoring time $C$, and the covariate $x$; the vector $\theta=\left(\psi_{1}^{\prime}, \psi_{2}^{\prime}, \phi^{\prime}\right)$ is the full vector of parameters where $\psi_{1}$ indexes the marginal recurrent event process, $\psi_{2}$ indexes the joint distribution of the marks, and $\phi$ characterizes the association between the marks and the event times. Conditional on $X=x$, with a sample of size $m$ and data $z=\left(z_{1}, \ldots, z_{m}\right)^{\prime}$, the maximum likelihood estimate $\widehat{\theta}$ is the solution to the score equation $U(\theta ; z)=\sum_{i=1}^{m} U\left(\theta ; z_{i}\right)=0$ where

$$
U\left(\theta ; z_{i}\right)=\frac{\partial \log L\left(\theta ; z_{i}\right)}{\partial \theta}
$$

and is consistent. Moreover,

$$
\sqrt{m}(\widehat{\theta}-\theta) \stackrel{D}{\longrightarrow} N\left(0, \mathcal{A}^{-1}(\theta) \mathcal{B}(\theta) \mathcal{A}^{-1}(\theta)\right)
$$

as $m \rightarrow \infty$, where $\mathcal{A}(\theta)=-E\left[\partial U\left(\theta ; Z_{i}\right) / \partial \theta^{\prime}\right]$ and $\mathcal{B}(\theta)=E\left[U\left(\theta ; Z_{i}\right) U^{\prime}\left(\theta ; Z_{i}\right)\right]$. If the model is correctly specified, $\mathcal{A}(\theta)=\mathcal{B}(\theta)$ and $\operatorname{asvar}(\sqrt{m}(\widehat{\theta}-\theta))=\mathcal{A}^{-1}(\theta)$. If the model is misspecified, the asymptotic covariance matrix has the more complex form $\mathcal{A}^{-1}(\theta) \mathcal{B}(\theta) \mathcal{A}^{-1}(\theta)$, which is the robust asymptotic covariance matrix that can provide protection from some forms of model misspecification. The robust covariance matrix is estimated by $\widehat{A}^{-1}(\widehat{\theta}) \widehat{B}(\widehat{\theta}) \widehat{A}^{-1}(\widehat{\theta})$, where

$$
\widehat{A}(\widehat{\theta})=-\left.\frac{1}{m} \sum_{i=1}^{m} \frac{\partial U\left(\theta ; z_{i}\right)}{\partial \theta}\right|_{\theta=\widehat{\theta}},
$$

and

$$
\widehat{B}(\widehat{\theta})=\left.\frac{1}{m} \sum_{i=1}^{m} U\left(\theta ; z_{i}\right) U^{\prime}\left(\theta ; z_{i}\right)\right|_{\theta=\hat{\theta}} .
$$

\section{Simulation Studies}

\subsection{Empirical Performance Under the Correct Copula}

Here we investigate the finite sample performance of maximum likelihood estimators based on the specified marked point process model and study empirically the relative efficiency of estimators from the joint model and corresponding marginal analyses of the recurrent event process and marks.

We first consider events generated from a joint process where the marginal model for the recurrent events corresponds to a non-homogeneous Poisson process as discussed in Section ??. The marginal rate function is of the form

$$
\lambda(t \mid X)=\alpha_{1} \alpha_{2}\left(\alpha_{1} t\right)^{\alpha_{2}-1} \exp (X \beta)
$$

giving the corresponding marginal mean function $E(N(t) \mid X)=\int_{0}^{t} \lambda(s \mid X) d s=\left(\alpha_{1} t\right)^{\alpha_{2}} \exp (X \beta)$. We set the administrative censoring time to $C_{A}=1, \alpha_{2}=0.75$ and $E(N(1) \mid X=0)=\alpha_{1}^{\alpha_{2}}=4$ giving $\alpha_{1}=6.35$. We set coefficient $\beta=\log (0.5)=-0.6931$ to indicate a $50 \%$ reduction of the risk of events under the experimental treatment. We assume that the marks are multivariate normal with $\sigma^{2}=1$ and consider marks with a moderate $(\rho=0.3)$ and strong correlation $(\rho=0.6)$. The means are assumed to be constant within groups with $\eta_{0 k}=\eta_{0}=0$ and $\eta_{1 k}=\eta_{1}=0.5, k=0,1, \ldots, K$ corresponding to an experimental treatment which gives a better mean response.

The event process and marks are linked through the Clayton copula,

$$
\mathcal{C}(u, v)=\left(u^{-\phi}+v^{-\phi}-1\right)^{-1 / \phi} \text { for } \phi>0 .
$$


We consider scenarios with both moderate (Kendall's $\tau=0.2)$ and strong $(\tau=0.6)$ dependence between the marks and the recurrent event process giving $\phi=2 \tau /(1-\tau)=0.5$ and 3 in (??) respectively (Nelsen, 2006). We consider $40 \%$ random right censoring with $P\left(C_{R}>C_{A}\right)=\exp \left(-\lambda_{c}\right)=$ 0.6 giving $\lambda_{c}=0.5108$. A total of 500 samples were simulated with 200 and 1000 subjects per sample for each parameter configuration. We next repeated the simulation study with events generated according to a negative binomial process with intensity function given by (??).

For each dataset, we carried out a "joint analysis" based on the copula-based marked point process model formulation described above with a Poisson margin $\left(\mathrm{JOINT}_{P}\right)$, where parameters are estimated by maximizing the likelihood in (??). Marginal analyses of the event times were also conducted based on a parametric Poisson model (NHPP) and a semi-parametric Andersen-Gill model (AG). Marginal analysis of the marks were also carried out based on generalized estimating equations (GEE) under both an independence working correlation structure (WI) and an exchangeable working correlation structure (EXCH). The empirical biases (BIAS), empirical standard error (ESE) and average standard error (ASE) are reported for each method of estimation and parameter configuration.

The BIAS of the estimators of $\log \left(\alpha_{1}\right), \log \left(\alpha_{2}\right)$ and $\beta$ are negligible for the parametric Poisson (NHPP) and joint analyses for all configurations with Poisson margin in Table ??, as is the case for the estimators of $\beta$ from the AG analysis. Moreover there is excellent agreement between the ESEs and average model-based standard errors. There is a marked decrease in the empirical and average model-based SEs of all estimators from the joint analysis compared to the marginal analyses, with the gain in efficiency greatest when $\tau$ is largest; not surprisingly there is little effect of the association between the marks on the relative efficiency.

The frequency properties of the estimates of the marks are reported in Table ??. The analysis of the marks based on GEEs with a working independence assumption yields heavily biased estimators; use of an exchangeable working correlation structure, which is compatible with the true structure, provides some protection against the selection effects arising from the association between the marks. The BIAS are smallest under the joint analysis and the respective estimates are most efficient as well.

In Table ?? we report the results for the marginal event process when data are generated from a joint model with a negative binomial intensity of the form (??) with $\gamma=0.5$. The resulting data were analysed under a misspecified parametric Poisson (NHPP), a semiparametric AG model, a semiparametric negative binomial model (SNB), a joint model with a misspecified Poisson event process $\left(\mathrm{JOINT}_{P}\right)$, and a joint model with a correctly specified negative binomial margin $\left(\mathrm{JOINT}_{N B}\right)$. In addition to reporting the empirical bias, ESE, and average model-based standard error, we also report the average robust standard error (RSE), calculated as described in the Section ??.

The biases are larger under the joint model with a Poisson margin revealing that misspecification of the marginal intensity is more of a concern in the joint analysis. There is generally good performance of the estimators under the correctly specified joint negative binomial model. The BIAS of the estimators of $\beta$ from the AG and semiparametric negative binomial analysis are negligible but robust standard errors are required for valid inferences in the former approach. Again there are appreciable gains in efficiency from the joint analysis with respect to most parameters with this gain being largest when $\tau$ is large.

The results from the marginal semiparametric analysis of the marks are given in Table ?? along with those from a joint analysis under Poisson and negative binomial assumptions for the marginal event process. The BIAS under GEE analysis with a working independence correlation structure are larger than those under an exchangeable correlation structure. Misspecification of the marginal model for the recurrent event process leads to biased estimators of the marginal parameters for the marks as well as estimation of the association parameter; this effect is stronger for larger $\tau$. Estimates of the fully and correctly specified joint model with a negative binomial margin have negligible empirical biases and the smallest standard errors compared to other valid approaches. 
Table 1: Frequency properties of parameter estimators for the recurrent event process through marginal and joint analysis of the marked point process with a Poisson margin under $40 \%$ random right censoring where $E(N(1) \mid X=0)=4 ; \alpha_{1}=6.35, \alpha_{2}=0.75, \beta=\log 0.5 ; \eta_{0}=0, \eta_{1}=0.5$, $\sigma^{2}=1 ; 500$ simulated samples

\begin{tabular}{|c|c|c|c|c|c|c|c|c|c|}
\hline \multirow[b]{2}{*}{$\rho$} & \multirow[b]{2}{*}{ Kendall's $\tau$} & & \multicolumn{2}{|c|}{$\log \left(\alpha_{1}\right)$} & \multicolumn{2}{|c|}{$\log \left(\alpha_{2}\right)$} & \multicolumn{3}{|c|}{$\beta$} \\
\hline & & & NHPP & $\mathrm{JOINT}_{P}$ & NHPP & $\mathrm{JOINT}_{P}$ & NHPP & AG & $\mathrm{JOINT}_{P}$ \\
\hline \multicolumn{10}{|c|}{ Results based on 200 subjects/sample } \\
\hline \multirow[t]{6}{*}{0.3} & 0.2 & BIAS & 0.0029 & 0.0027 & 0.0011 & 0.0012 & -0.0022 & -0.0021 & -0.0023 \\
\hline & & ESE & 0.1039 & 0.1005 & 0.0428 & 0.0415 & 0.0943 & 0.0942 & 0.0911 \\
\hline & & ASE & 0.1037 & 0.1015 & 0.0431 & 0.0420 & 0.0965 & 0.0965 & 0.0929 \\
\hline & 0.6 & BIAS & -0.0022 & -0.0004 & 0.0035 & 0.0023 & 0.0015 & 0.0015 & 0.0004 \\
\hline & & ESE & 0.1052 & 0.0891 & 0.0431 & 0.0306 & 0.0956 & 0.0955 & 0.0764 \\
\hline & & ASE & 0.1033 & 0.0871 & 0.0431 & 0.0304 & 0.0964 & 0.0964 & 0.0726 \\
\hline \multirow[t]{6}{*}{0.6} & 0.2 & BIAS & 0.0029 & 0.0026 & 0.0011 & 0.0012 & -0.0022 & -0.0021 & -0.0020 \\
\hline & & ESE & 0.1039 & 0.1011 & 0.0428 & 0.0417 & 0.0943 & 0.0942 & 0.0905 \\
\hline & & ASE & 0.1037 & 0.1017 & 0.0431 & 0.0421 & 0.0965 & 0.0965 & 0.0926 \\
\hline & 0.6 & BIAS & -0.0022 & -0.0017 & 0.0035 & 0.0026 & 0.0015 & 0.0015 & 0.0012 \\
\hline & & ESE & 0.1052 & 0.0904 & 0.0431 & 0.0325 & 0.0956 & 0.0955 & 0.0725 \\
\hline & & ASE & 0.1033 & 0.0888 & 0.0431 & 0.0324 & 0.0964 & 0.0964 & 0.0690 \\
\hline \multicolumn{10}{|c|}{ Results based on 1000 subjects/sample } \\
\hline \multirow[t]{6}{*}{0.3} & 0.2 & BIAS & -0.0014 & -0.0011 & 0.0014 & 0.0012 & 0.0019 & 0.0019 & 0.0012 \\
\hline & & ESE & 0.0461 & 0.0454 & 0.0191 & 0.0185 & 0.0447 & 0.0447 & 0.0429 \\
\hline & & ASE & 0.0463 & 0.0454 & 0.0193 & 0.0188 & 0.0430 & 0.0430 & 0.0414 \\
\hline & 0.6 & BIAS & -0.0007 & -0.0013 & 0.0018 & 0.0014 & 0.0008 & 0.0008 & 0.0012 \\
\hline & & ESE & 0.0461 & 0.0380 & 0.0191 & 0.0135 & 0.0430 & 0.0430 & 0.0320 \\
\hline & & ASE & 0.0463 & 0.0390 & 0.0193 & 0.0136 & 0.0429 & 0.0429 & 0.0323 \\
\hline \multirow[t]{6}{*}{0.6} & 0.2 & BIAS & -0.0014 & -0.0012 & 0.0014 & 0.0012 & 0.0019 & 0.0019 & 0.0011 \\
\hline & & ESE & 0.0461 & 0.0456 & 0.0191 & 0.0186 & 0.0447 & 0.0447 & 0.0427 \\
\hline & & ASE & 0.0463 & 0.0455 & 0.0193 & 0.0188 & 0.0430 & 0.0430 & 0.0413 \\
\hline & 0.6 & BIAS & -0.0007 & -0.0015 & 0.0018 & 0.0015 & 0.0008 & 0.0008 & 0.0011 \\
\hline & & ESE & 0.0461 & 0.0391 & 0.0191 & 0.0143 & 0.0430 & 0.0430 & 0.0303 \\
\hline & & ASE & 0.0463 & 0.0398 & 0.0193 & 0.0145 & 0.0429 & 0.0429 & 0.0308 \\
\hline
\end{tabular}

NHPP denotes a nonhomogeneous Poisson process model with rate $\lambda\left(t \mid H^{N}(t), X\right)=\alpha_{1} \alpha_{2}\left(\alpha_{1} t\right)^{\alpha_{2}-1} \exp (\beta X)$, AG denotes a semiparametric analysis with $\lambda\left(t \mid H^{N}(t), X\right)=\lambda_{0}(t) \exp (\beta X)$ with $\lambda_{0}(t)$ unspecified, and JOINT $_{P}$ corresponds to the joint model with Poisson margin for the event intensity. 


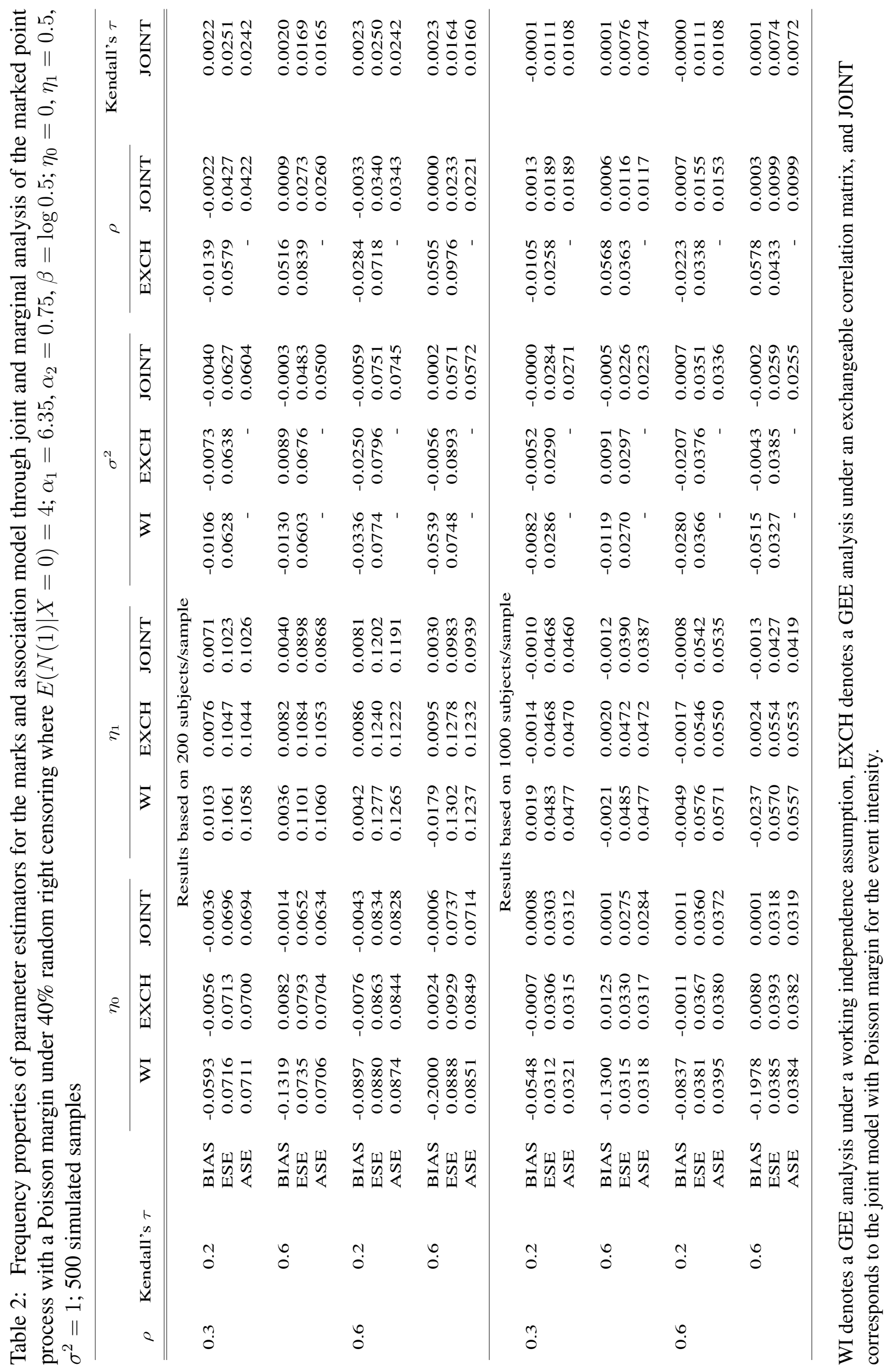




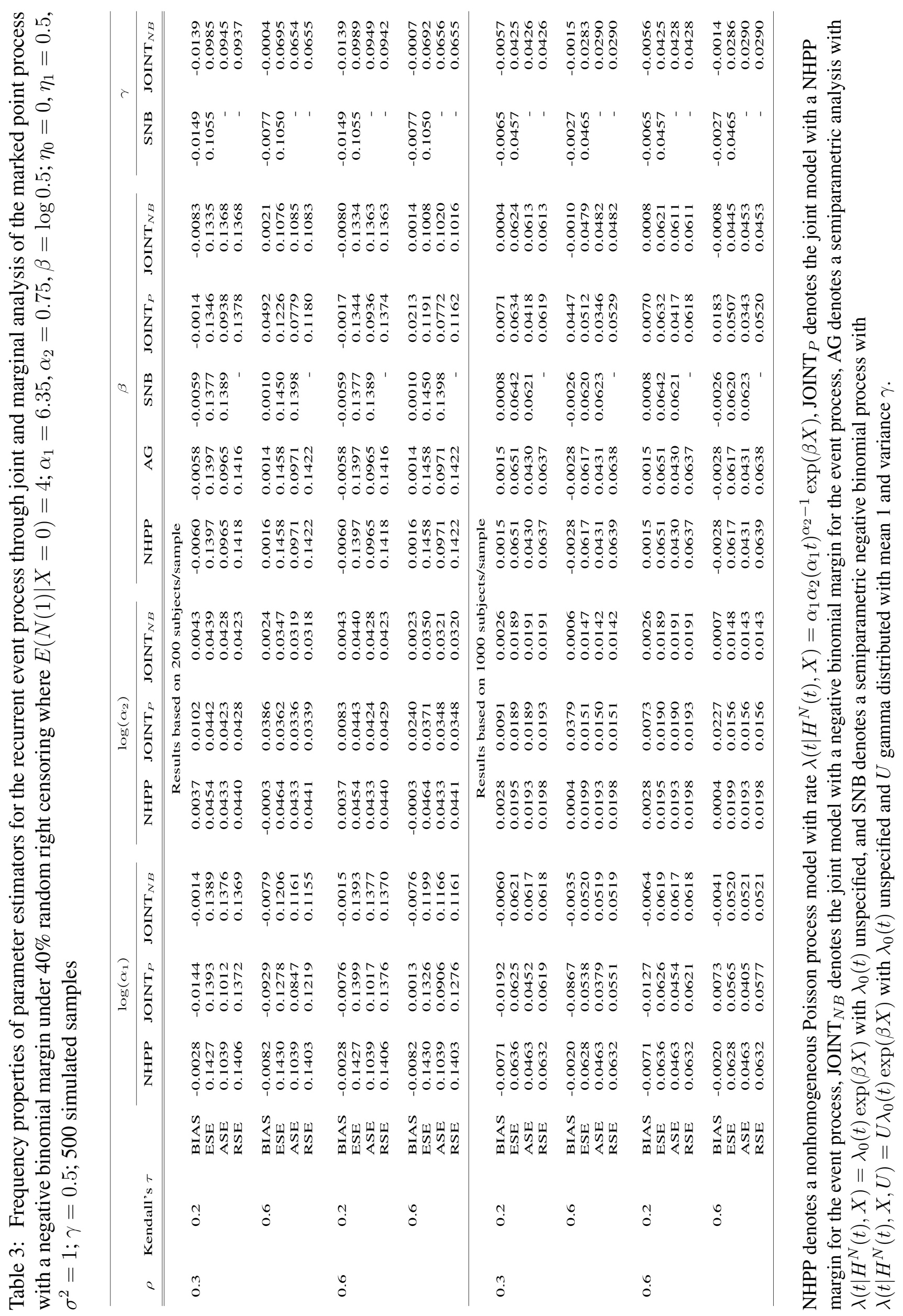




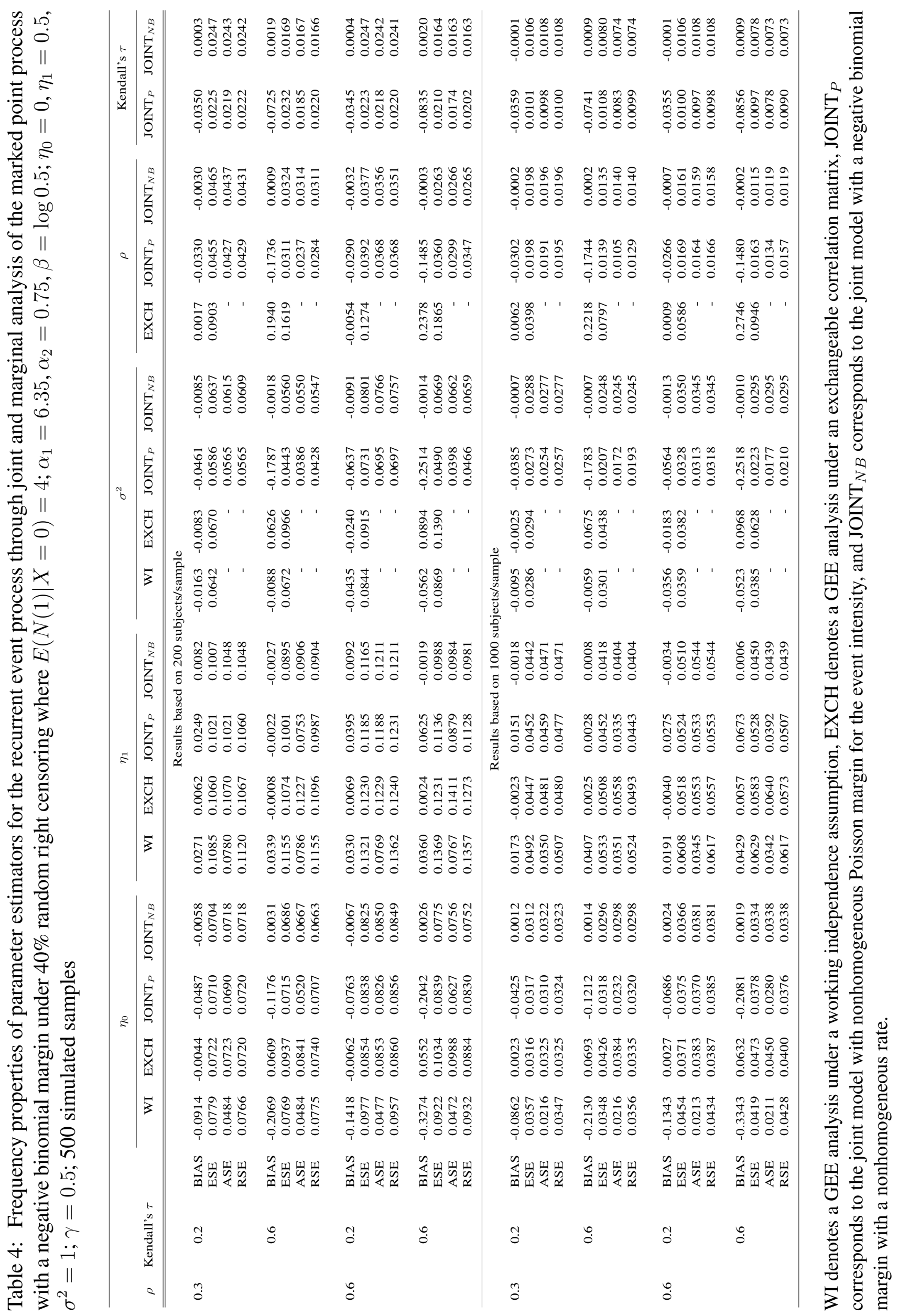




\subsection{EMPIRICAl PERFormance Under COPUla MisSPECIFICATION}

Prokhorov and Schmidt (2009) discuss asymptotic properties of parameter estimates under misspecification of the copula model and characterize settings in which inferences can be robust regarding features of the marginal distributions. Other authors have examined the issue of copula misspecification in some special cases. Chatterjee et al. (2006) investigate misspecification in the context of "parallel" bivariate survival data in response-dependent (i.e. case-control and case-only) designs and find misspecification of the copula function does not lead to appreciable bias in estimators of the marginal parameters for some types of misspecification. Craiu and Craiu (2008) empirically study the effect of copula misspecification on conditional means and variances and show there can be significant impact on inferences; careful consideration of the copula family is therefore required.

Here we deal with a setting involving life history data in which the copula plays a role in defining the conditional distribution of successive marks and gap times. To provide insight into the effect of misspecification in this context, we carried out further simulations in which data were generated using a Frank copula and a Gumbel-Hougaard copula, both of which are in the Archimedian family (Nelson, 2006); analyses, however, were conducted based on the Clayton copula. We considered $\rho=0.3$ and 0.6 for the correlations between the marks, and $\tau=0.2$ and 0.6 for the dependence between the marks and the gap times, and restricted attention to the setting in which the events were generated according to a marginal Poisson process and with a sample size of $m=1000$.

The results are reported in Table 5 and reveal that biases arising when the copula is misspecified are negligible for the marginal parameters when Kendall's $\tau$ is modest, but can be appreciable when Kendall's $\tau$ is large. Not surprisingly the biases for Kendall's $\tau$ are generally quite large. We remark that in addition to biases that may arise due to copula misspecification in the context of "parallel" bivariate survival data, the limiting behaviour in our context depends on the validity of the likelihood in (??) and specifically the conditional independence assumptions A1 and A2, which are not satisfied under misspecification of the copula.

\section{An Illustration Application to Data from the Mirasol Study}

The Mirasol Study (Cazenave et al., 2010) is a multicenter, open-label, parallel-group non-inferiority randomized controlled trial in which 118 haematology /oncology patients with thrombocytopenia were randomized to receive either a pathogen-reduced platelet product (PRT-PLT) $(X=1)$ or standard reference platelets $(X=0)$ as required over a 28 -day treatment period (i.e. $C_{A}=28$ ). The primary outcome was transfusion-based and defined as the 24-hour corrected count increment (CCI). As mentioned earlier, the count increment is the post-transfusion platelet count measured $24 \mathrm{~h}$ after transfusion minus the pre-transfusion platelet count and the CCI adjusts this number by the dose of platelets transfused and the patients' body surface area (Davis et al., 1999).

In Table ?? we report on analyses of the Mirasol Study based on separate marginal and joint analyses for the events and marks as discussed in Section ??. The estimate of Kendall's $\tau$ from the joint model with the negative binomial margin is 0.157 (s.e. $(\widehat{\tau})=0.032, p<0.001$ ), so there is significant but modest dependence between the CCI at the $k$ th transfusion and the time to the next transfusion $\left(W_{k+1}=T_{k+1}-T_{k}\right)$. We therefore may not expect an appreciable gain in efficiency in this dataset from the joint modeling.

The model-based standard errors for $\widehat{\beta}$ from the parametric and semiparametric Poisson analyses are smaller than the respective RSEs, which in turn are close to the model-based standard error of $\widehat{\beta}$ from the semiparametric negative binomial model. This suggests the presence of extra-Poisson variation; the likelihood ratio test statistic of $H_{0}: \gamma=0$ versus $H_{A}: \gamma>0$ asymptotically follows a distribution which is a $50: 50$ mixture of a point mass at zero and a $\chi_{1}^{2}$ distribution (Self and Liang, 1987). The resulting test statistic is 10.344 which gives a $\mathrm{p}-$ value of $0.5 P\left(\chi_{1}^{2}>10.344\right)=0.0007$ 
Table 5: Sensitivity analysis on the effect of copula misspecification showing the frequency properties of estimators via joint analysis of the marked point process ; marginal intensity is Poisson; $40 \%$ right censoring with $E(N(1) \mid X=0)=4 ; \alpha_{1}=6.35, \alpha_{2}=0.75, \beta=\log 0.5 ; \eta_{0}=0, \eta_{1}=0.5$, $\sigma^{2}=1 ; 1000$ subjects/sample; 500 simulated samples

\begin{tabular}{|c|c|c|c|c|c|c|c|c|c|c|}
\hline$\rho$ & Kendall's $\tau$ & & $\log \left(\alpha_{1}\right)$ & $\log \left(\alpha_{2}\right)$ & $\beta$ & $\eta_{0}$ & $\eta_{1}$ & $\sigma^{2}$ & $\rho$ & Kendall's $\tau$ \\
\hline \multicolumn{11}{|c|}{ Data are generated using Frank copula } \\
\hline \multirow[t]{6}{*}{0.3} & 0.2 & BIAS & 0.0046 & -0.0026 & 0.0054 & -0.0022 & -0.0049 & 0.0115 & 0.0012 & -0.0427 \\
\hline & & ESE & 0.0488 & 0.0202 & 0.0432 & 0.0311 & 0.0495 & 0.0278 & 0.0193 & 0.0112 \\
\hline & & ASE & 0.0460 & 0.0190 & 0.0420 & 0.0316 & 0.0468 & 0.0276 & 0.0189 & 0.0112 \\
\hline & 0.6 & BIAS & 0.0555 & -0.0302 & 0.0635 & -0.0326 & -0.0468 & 0.0809 & 0.0079 & -0.0810 \\
\hline & & ESE & 0.0439 & 0.0152 & 0.0409 & 0.0320 & 0.0486 & 0.0297 & 0.0164 & 0.0098 \\
\hline & & ASE & 0.0423 & 0.0151 & 0.0347 & 0.0307 & 0.0435 & 0.0267 & 0.0140 & 0.0085 \\
\hline \multirow[t]{6}{*}{0.6} & 0.2 & BIAS & 0.0045 & -0.0026 & 0.0053 & -0.0021 & -0.0055 & 0.0132 & 0.0014 & -0.0427 \\
\hline & & ESE & 0.0490 & 0.0203 & 0.0431 & 0.0377 & 0.0571 & 0.0345 & 0.0157 & 0.0113 \\
\hline & & ASE & 0.0460 & 0.0190 & 0.0419 & 0.0379 & 0.0546 & 0.0344 & 0.0154 & 0.0112 \\
\hline & 0.6 & BIAS & 0.0632 & -0.0346 & 0.0586 & -0.0439 & -0.0402 & 0.0965 & 0.0114 & -0.0800 \\
\hline & & ESE & 0.0458 & 0.0163 & 0.0389 & 0.0392 & 0.0548 & 0.0379 & 0.0142 & 0.0098 \\
\hline & & ASE & 0.0433 & 0.0158 & 0.0334 & 0.0357 & 0.0483 & 0.0321 & 0.0115 & 0.0084 \\
\hline \multicolumn{11}{|c|}{ Data are generated using Gumbel-Hougaard copula } \\
\hline \multirow[t]{6}{*}{0.3} & 0.2 & BIAS & 0.0073 & -0.0050 & 0.0077 & -0.0015 & -0.0054 & 0.0111 & -0.0000 & -0.0527 \\
\hline & & ESE & 0.0468 & 0.0191 & 0.0426 & 0.0311 & 0.0469 & 0.0282 & 0.0197 & 0.0126 \\
\hline & & ASE & 0.0462 & 0.0191 & 0.0421 & 0.0316 & 0.0468 & 0.0277 & 0.0188 & 0.0114 \\
\hline & 0.6 & BIAS & 0.0739 & -0.0389 & 0.0570 & -0.0418 & -0.0391 & 0.0966 & 0.0089 & -0.0897 \\
\hline & & ESE & 0.0458 & 0.0164 & 0.0415 & 0.0340 & 0.0496 & 0.0303 & 0.0161 & 0.0095 \\
\hline & & ASE & 0.0430 & 0.0154 & 0.0351 & 0.0311 & 0.0442 & 0.0275 & 0.0143 & 0.0086 \\
\hline \multirow[t]{6}{*}{0.6} & 0.2 & BIAS & 0.0073 & -0.0050 & 0.0074 & -0.0015 & -0.0049 & 0.0128 & 0.0008 & -0.0526 \\
\hline & & ESE & 0.0470 & 0.0193 & 0.0424 & 0.0369 & 0.0531 & 0.0358 & 0.0157 & 0.0127 \\
\hline & & ASE & 0.0463 & 0.0191 & 0.0420 & 0.0379 & 0.0547 & 0.0344 & 0.0154 & 0.0114 \\
\hline & 0.6 & BIAS & 0.0834 & -0.0442 & 0.0527 & -0.0563 & -0.0310 & 0.1133 & 0.0121 & -0.0886 \\
\hline & & ESE & 0.0470 & 0.0176 & 0.0392 & 0.0409 & 0.0552 & 0.0381 & 0.0136 & 0.0095 \\
\hline & & ASE & 0.0442 & 0.0161 & 0.0339 & 0.0363 & 0.0492 & 0.0330 & 0.0117 & 0.0085 \\
\hline
\end{tabular}




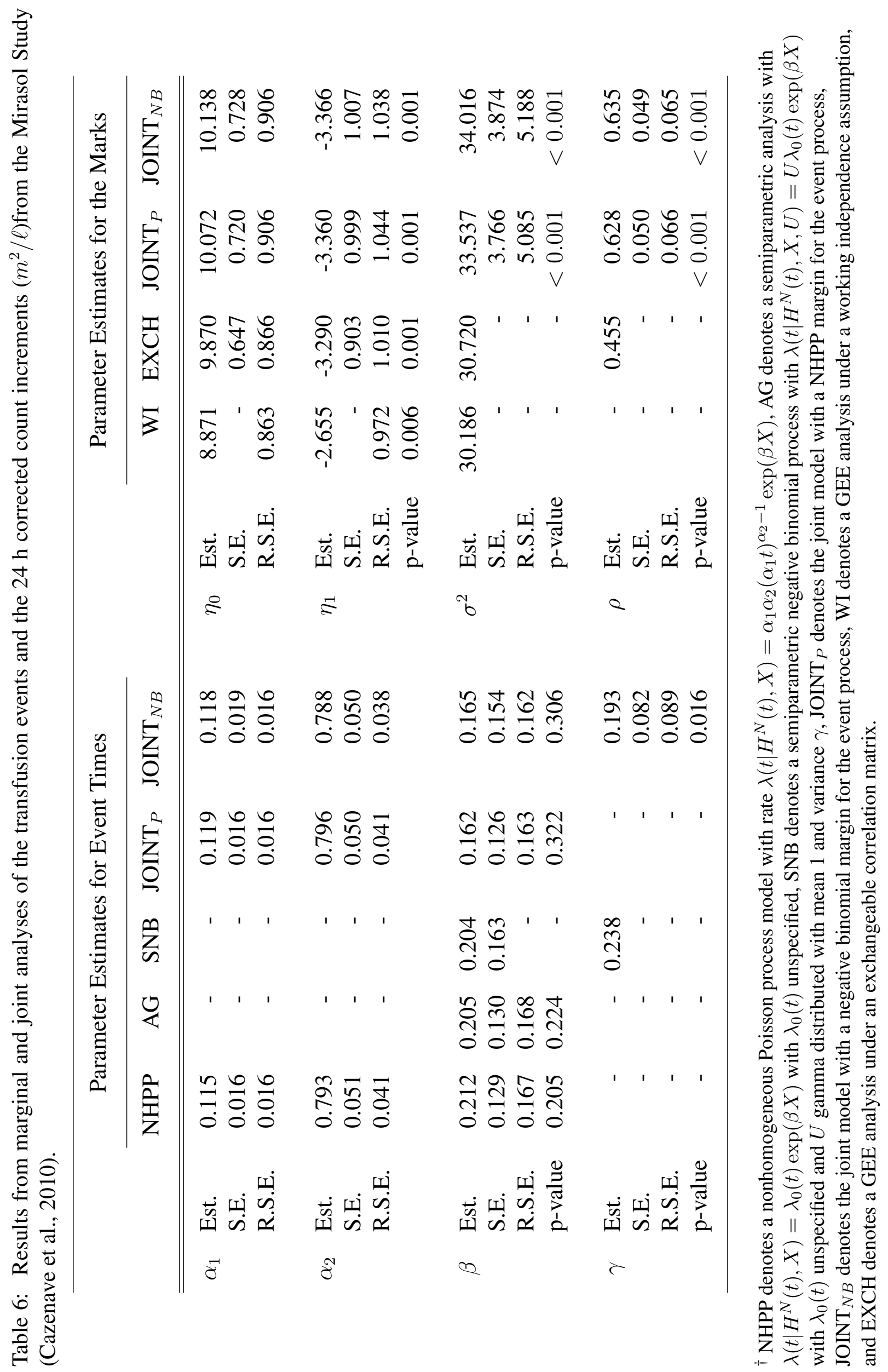


and rejection of the Poisson assumption for the marginal model. The estimated treatment effect on the event rate is $R R=1.18$ (95\% CI: $0.86,1.62 ; p=0.306)$ reflecting a nonsignificant trend towards increased need for transfusion in the PRT-PLT arm.

Regarding the marks, there is a consistent finding of a significantly lower response in the PRT-PLT arm compared to the reference arm. In the joint analysis with the negative binomial margin, we see a lower response by on average $3.37(95 \% \mathrm{CI}:-5.40,-1.33 ; p=0.001) \times 10^{9} \mathrm{~m}^{2} / \ell$. Thus noninferiority of the PRT-PLT product was not successfully demonstrated based on the 24-h CCI response. The correlation between the marks is quite strong at 0.635 from the joint negative binomial analysis suggesting the importance of accommodating this association in the joint model for the marks.

Figure ?? contains plots of the estimated mean function from a parametric analysis with a nonhomogeneous baseline Poisson rate function, a semiparametric Andersen-Gill analysis, and a joint analysis based on a Poisson and negative binomial intensity function. There is excellent agreement between the three parametric analyses and close agreement between all four in terms of the expected number of transfusions required over the 28 day treatment period. The estimated mean function based on the semiparametric AG model reflects a higher rate during the first two weeks followed by a lower rate in the latter two weeks; this is not exhibited in the parametric models suggesting the need for more flexible piecewise constant baseline rate functions.
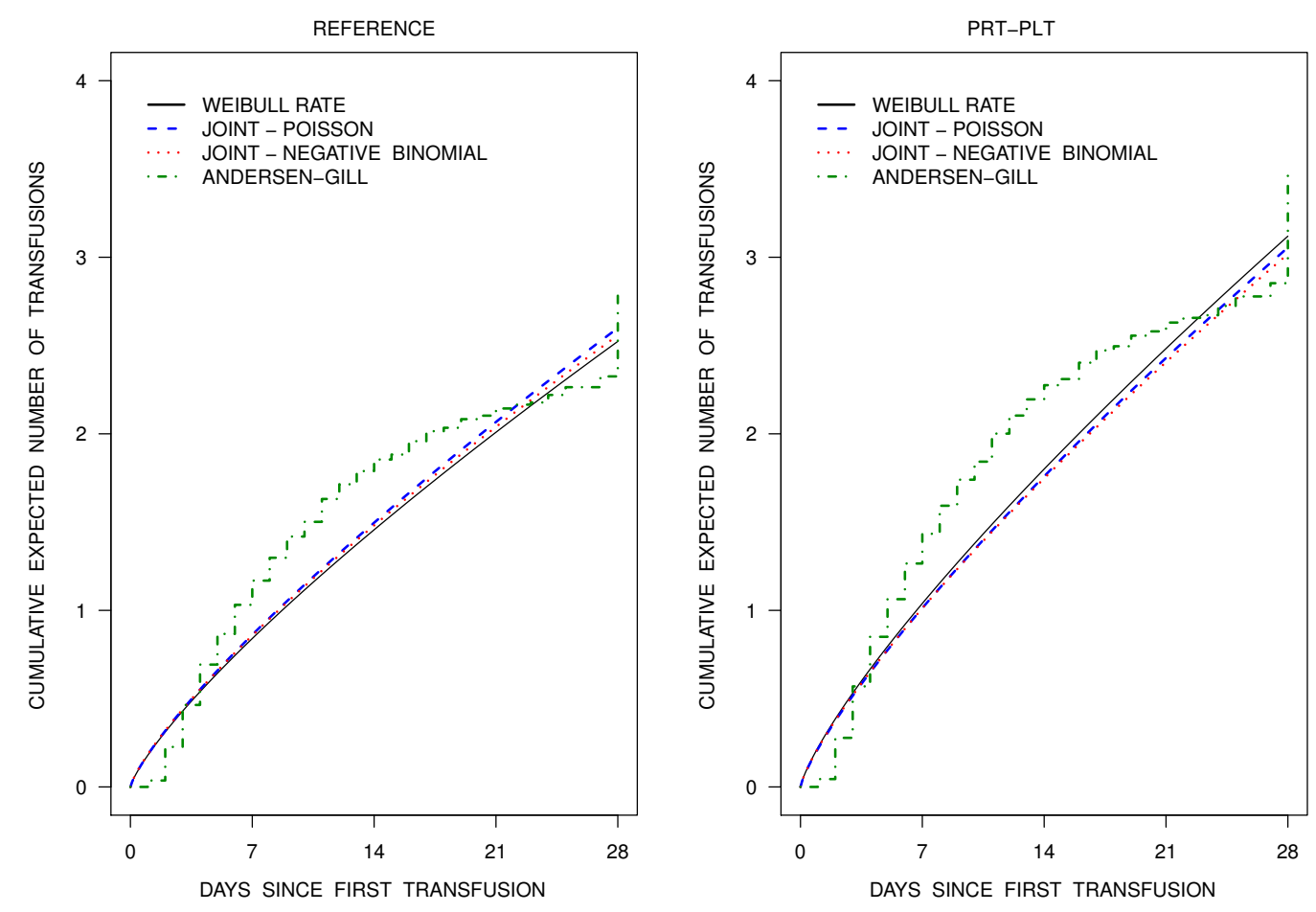

Figure 3: Plots of estimated baseline mean function obtained by integrating the marginal rate from a marginal analysis under a Poisson assumption with a parametric Weibull rate and semiparametric Andersen-Gill and from a joint analysis using (??) with a Poisson and negative binomial parametric marginal rate

\section{Discussion}

The idea of using copula functions to link the marks and the event times has been proposed in the ruin theory literature based on renewal processes (Albrecher and Teugels, 2006; Landriault et al., 2013) 
but these models have different formulations motivated by issues arising in actuarial science. This model is the first we are aware of that address an important problem prevalent in the health research.

The model we proposed in Section ?? is a fully parametric model and it would be desirable to relax the parametric assumption for the baseline rate within the class of mixed Poisson marginal processes to obtain some robustness. A simple first step would be to assume a piecewise constant baseline rate which would require specification of break points at which the rate can change. This approach has the advantage that it facilitates parametric analysis with a more flexible form for the baseline rate and it can be shown to give good approximations to results from semiparametric models. A disadvantage is the need to specify the break points and the arbitrary nature of any choices one might make for them. Recent work by Lawless and Yilmaz (2011a,b) on fitting bivariate failure time data with marginal Cox models and a specified copula suggests that some progress could be made in semiparametric analysis. Lawless and Yilmaz (2011a) comment that the two-stage semiparametric estimator of the copula parameter is about as good as the semiparametric simultaneous estimator of it. Since there are considerable computational challenges that arise in a joint analysis, two-stage estimation procedures (Shih and Louis, 1995) can be applied in principle, however, there will always be a trade-off between the need to make greater assumptions in the joint analysis with the robustness to dependent observation schemes that arise from joint analysis. A compromise approach would be to consider a two-stage procedure in which only the event process model is fit at the first stage. The resulting estimates could then be inserted into (??) and maximization could be carried out with respect to the marginal parameters of the marks and the association parameters in the copula. This would permit use of semiparametric estimates of the event process obtained by standard methods, and would provide protection against the dependent observation scheme in the modeling of the marks at the second stage. This approach is currently under investigation.

Several other extensions are possible to this model. We assumed that the dependence between the recurrent event process and the marks remains unchanged for each consecutive pair of marks and waiting times. We also assumed that the association between the mark and the subsequent waiting time was the same in the two treatment arms (??). One could generalize the model to allow different copula parameters or even different copula functions for successive pairs of marks and waiting times, and these could even differ between the two treatment arms.

We have empirically quantified the efficiency gains that can be realized from joint analyses. We also studied the effect of misspecifying the marginal intensity for event times under the assumption that the copula function was correctly specified. We also examined empirical properties of estimators arising from misspecification of the copula function and found, in our limited investigation, only modest impact when Kendall's $\tau$ is modest. When the association between the marks and subsequent waiting times is strong, the copula model should be checked carefully. One attractive avenue for doing this is through model expansion; see Yilmaz and Lawless (2011) for a discussion of this approach in the failure time context.

\section{ACKNOWLEDGEMENTS}

This research was supported by Grants from the Natural Sciences and Engineering Research Council of Canada (RGPIN 155849) and the Canadian Institutes for Health Research (FRN 13887). Richard Cook is a Canada Research Chair in Statistical Methods for Health Research. The authors thank Professor Jerry Lawless and Professor Nancy Heddle for helpful discussions and collaboration and Ray Goodrich for helpful discussion and permission to use the data from the Mirasol Study. 


\section{REFERENCES}

Aalen, O.O., Borgan, O., and Gjessing, H.K. (2008). Survival and Event History Analysis: A Point Process Point of View. Springer, New York.

Albrecher, H., and Teugels, J.L.(2006). Exponential behavior in the presence of dependence in risk theory. Journal of Applied Probability 43, 257-273.

Andersen, P.K., and Gill, R.D. (1982). Cox's regression model for counting processes: A large sample study. The Annals of Statistics 10, 1100-1120.

Andersen, P.K., Borgan, O., Gill, R.D., and Keiding, N. (1993). Statistical Models Based on Counting Processes. Springer-Verlag, New York.

Cazenave, J-P, Folléa, G., Bardiaux, L., Boiron, J-M, Lafeuillade, B., Debost, M., Lioure, B., Harousseau, J-L, Tabrizi, R., Cahn, J-Y, Michallet, M., Ambruso, D., Schots, R., Tissot, J-D, Sensebé, L., Kondo, T., McCullough, J., Rebulla, P., Escolar, G., Mintz, P., Heddle, N.M., Goodrich, R.P., Bruhwyler, J., Le, C., Cook, R.J., Stouch, B., for members of the The Mirasol Clinical Evaluation Study Group. (2010). A randomized controlled clinical trial evaluating the performance and safety of platelets treated with MIRASOL pathogen reduction technology. Transfusion 50, 2362-2375.

Chatterjee, N., Kalaylioglu, Z., Shih, J.H., and Gail, M.H. (2006). Case-control and case-only designs with genotype and family history data: estimating relative risk, residual familial aggregation, and cumulative risk. Biometrics 62, 36-48.

Chen, F., and Zheng, Y-S. (1997). One-warehouse multiretailer systems with centralized stock information. Operations Research 45, 275-287.

Cole, E.H., Cattran, D.C., Farewell, V.T., Aprile, M., Bear, R.A., Pei, Y.P., Fenton, S.S., L. Tober, J.A., Cardella, C.J. (1994). A comparison of rabbit antithymocyte serum ane okt3 as prophylaxis against renal allograft rejection. Transplantation 57, 60-67.

Cook, R.J., Lawless, J.F., and Lee, K.-A. (2003). Cumulative processes related to event histories. SORT 27, 13-30.

Cook, R.J., and Lawless, J.F. (2007). The Statistical Analysis of Recurrent Events. Springer Science + Business Media, LLC, New York.

Cox, D.R., and Isham, V. (1980). Point Processes. Chapman and Hall, London.

Craiu, M. and Craiu, R.V. (2008). Choice of parametric families of copulas. Advances and Applications in Statistics 10, 25-40.

Daley, D.J., and Vere-Jones, D. (2008). An Introduction to the Theory of Point Processes: Volumn II: General Theory and Structure, 2nd Edition. Springer, New York.

Davis, K.B., Slichter, S.J., and Corash, L. (1999). Corrected count increment and percent platelet recovery as measures of post-transfusion platelet response: problems and a solution. Transfusion 39, $586-592$.

Descombes, X., and Zerubia, J. (2002). Marked point process in image analysis. Signal Processing Magazine, IEEE 19, 77-84.

Fok C.C.T., Ramsay J.O., Abrahamowicz, M., and Fortin, P. (2012). A functional marked point process model for lupus data. Canadian Journal of Statistics 40, 517-529. 
Genest, C., and MacKay, J. (1986). The joy of copulas: bivariate distributions with uniform marginals. The American Statistician 40, 280-283.

Goulard, M., Säkk ä, A., and Grabarnik, P. (1996). Parameter estimation for marked Gibbs point processes through the maximum pseudo-likelihood method. Scandinavian Journal of Statistics 23, $365-379$.

Grandell, J. (1997). Mixed Poisson Processes. Chapman and Hall, London.

Guan, Y. (2006). Tests for independence between marks and points of a marked point process. Biometrics 62, 126-134.

Holden, L., Sannan, S., and Bungum, H. (2002). A stochastic marked point process model for earthquakes. Natural Hazards and Earth System Science 3, 95-101.

Joe, H. (1997). Multivariate Dependence Concepts, Chapman and Hall, London.

Kalbfleisch, J.D., and Prentice, R.L. (2002). The Statistical Analysis of Failure Time Data, 2nd Edition. John Wiley and Sons, New York.

Karr, A. F. (1991). Point Processes and Their Statistical Inference 2nd edition. Dekker, New York.

Landriault, D., Lee, W.Y., Willmot, G.E., and Woo J.-K. (2013). A note on deficit analysis in dependency models involving Coxian claim amounts. Scandinavian Journal of Statistics, DOI:10.1080/03461238.2012.723044

Lawless, J.F. (1987a). Negative binomial and mixed Poisson regression. Canadian Journal of Statistics 15, 209-225.

Lawless, J.F. (1987b). Regression methods for Poisson process data. Journal of the American Statistical Association 82, 808-815.

Lawless, J.F. (2003). Statistical Models and Methods for Lifetime Data, 2nd edition. Wiley, Hoboken, NJ.

Lawless, J.F., and Nadeau, J.C. (1995). Nonparametric estimation of cumulative mean functions for recurrent events. Technometrics 37, 158-168.

Lawless, J.F., and Yilmaz, Y.E. (2011a). Comparison of semiparametric maximum likelihood estimation and two-stage semiparametric estimation in copula models. Computational Statistics and Data Analysis 55, 2446-2455.

Lawless, J.F., and Yilmaz, Y.E. (2011b). Semiparametric estimation in copula models for bivariate sequential survival times. Biometrical Journal 53, 779-796.

Nelsen, R.B. (2006). An Introduction to Copulas, Springer, New York.

Pascual, J., Falk, R.M., Piessens, F., Prusinski, A., Docekal, P., Robert, M., Ferrer, P., Luria, X., Segarra, R., and Zayas, J.M. (2000). Consistent efficacy and tolerability of almotriptan in the acute treatment of multiple migraine attacks: results of a large, randomized, double-blind, placebocontrolled study. Cephelegia 20, 588-596.

Patton, A.J. (2006). Modelling asymmetric exchange rate dependence. International Economic Review 47, 527-556. 
Penttinen, A., Stoyan, D., and Henttonen, H.M. (1992). Marked point processes in forest statistics Forest Science 38, 806-824.

Petri, M., Genovese, M., Engle, E., and Hochberg, M. (1991). Definition, incidence, and clinical description of flare in systemic lupus erythematosus. A prospective cohort study. Arthritis \& Rheumatism 34, 937-944.

Politis, D.N., and Sherman M. (2001). Moment Estimation for Statistics from Marked Point Processes. Journal of the Royal Statistical Society, Series B 63, 261-275.

Prentice, R.L., Williams, B.J., and Peterson, A.V. (1981). On the regression analysis of multivariate failure time data. Biometrika 68, 373-379.

Prigent, J-L. (2001). Option pricing with a general marked point process. Mathematics of Operations Research 26, 50-66.

Prokhorov, A. and Schmidt, P. (2009). Likelihood-based estimation in a panel setting: robustness, redundancy and validity of copulas. Journal of Econometrics 153, 93-104.

Robin, S. (2002). A compound Poisson model for word occurrences in DNA sequences. Journal of the Royal Statistical Society, Series C 51, 437-451.

Schlather, M., Ribeiro, P.J.Jr., and Diggle, P.J. (2004). Detecting dependence between marks and locations of marked point processes. Journal of the Royal Statistical Society, Series B 66, 79-93.

Schoenberg, F.P. (2004). Testing separability in spatialtemporal marked point processes. Biometrics 60, 471-481.

Sears, M.R., Taylor, D.R., Print, C.G., Lake, D.C., Li, Q., Flannery, M., Yates, D.M., Lucas, M.K., and Herbison, G.P. (1990). Regular inhaled beta-agonist treatment in bronchial asthma. Lancet 336, 1391-1396.

Self, S.G. and Liang, K.-Y. (1987). Asymptotic properties of maximum likelihood estimators and likelihood ratio tests under nonstandard conditions. Journal of the American Statistical Association 82, 605-610.

Shih, J.H., and Louis, T.A. (1995). Inferences on the association parameters in copula models for bivariate survival data. Biometrics 51, 1384-1399.

Snyder, D.L., and Miller, M.I. (1991). Random Point Processes in Time and Space. Springer-Verlag, New York.

Verona, E., Petrov, D., Cserhati, E., Hofman, J., Geppe, N., Medley, H., and Hughes, S. (2003). Fluticasone propionate in asthma: A long term dose comparison study. Archives of Disease in Childhood 88, 503-509.

Wei, L.J., Lin, D.Y., and Weissfeld, L. (1989). Regression analysis of multivariate incomplete failure time data by modeling marginal distributions. Journal of the American Statistical Association 84, 1065-1073.

Yilmaz, Y.E. and Lawless, J.F. (2011). Likelihood ratio procedures and tests of $\mathrm{t}$ in parametric and semiparametric copula models with censored data. Lifetime Data Analysis 17, 386-408. 